\title{
On the Double Roman Domination Number of Generalized Sierpiński Graphs
}

\author{
Anu V.* \\ Department of Mathematics \\ St.Peter's College \\ Kolenchery - 682311 \\ Kerala, India.
}

\author{
Aparna Lakshmanan S. ${ }^{\dagger}$ \\ Department of Mathematics \\ St.Xavier's College for Women \\ Aluva - 683101 \\ Kerala, India.
}

\begin{abstract}
In this paper, we study the double Roman domination number of generalized Sierpiński graphs $S(G, t)$. More precisely, we obtain a bound for the double Roman domination number of $S(G, t)$. We also find the exact value of $\gamma_{d R}\left(S\left(K_{n}, 2\right)\right)$.
\end{abstract}

Keywords: Double Roman Dominating Function, Double Roman Domination Number, Sierpiński Graphs.

AMS Subject Classification: 05C69; 05C76

\section{Introduction}

Let $G=(V(G), E(G))$ be a graph with vertex set $V(G)$ and edge set $E(G)$. If there is no ambiguity in the choice of $G$, then we write $V(G)$ and $E(G)$ as $V$ and $E$, respectively. Let $f: V \rightarrow\{0,1,2,3\}$ be a function defined on $V(G)$. Let $V_{i}^{f}=\{v \in V(G): f(v)=i\}$. (If there is no ambiguity, $V_{i}^{f}$ is written as $V_{i}$.) Then $f$ is a double Roman dominating function (DRDF) on $G$ if it satisfies the following conditions.

(i) If $v \in V_{0}$, then vertex $v$ must have at least two neighbors in $V_{2}$ or at least one

\footnotetext{
*E-mail : anusaji1980@gmail.com
}

${ }^{\dagger}$ E-mail : aparnaren@gmail.com 
neighbor in $V_{3}$.

(ii) If $v \in V_{1}$, then vertex $v$ must have at least one neighbor in $V_{2} \cup V_{3}$.

The weight of a DRDF $f$ is the sum $f(V)=\sum_{v \in V} f(v)$. The double Roman domination number, $\gamma_{d R}(G)$, is the minimum among the weights of DRDFs on $G$, and a DRDF on $G$ with weight $\gamma_{d R}(G)$ is called a $\gamma_{d R^{-}}$function of $G$ [6].

Let $\left(V_{0}, V_{1}, V_{2}, V_{3}\right)$ be the ordered partition of $V$ induced by $f$. Note that there exists a $1-1$ correspondence between the functions $f$ and the ordered partitions $\left(V_{0}, V_{1}, V_{2}, V_{3}\right)$ of $V$. Thus we will write $f=\left(V_{0}, V_{1}, V_{2}, V_{3}\right)$.

R. A. Beeler, T. W. Haynes and S. T. Hedetniemi pioneered the study of double Roman domination in [6]. The relationship between double Roman domination and Roman domination and the bounds on the double Roman domination number of a graph $G$ in terms of its domination number were discussed by them. They also determined a sharp upper bound on $\gamma_{d R}(G)$ in terms of the order of $G$ and characterized the graphs attaining this bound. In [1, it was verified that the decision problem associated with $\gamma_{d R}(G)$ is NP-complete for bipartite and chordal graphs. Above all this, a characterization of graphs $G$ with small $\gamma_{d R}(G)$ was provided. In [8], G. Hao et al. introduced the study of the double Roman domination of digraphs and L. Volkmann proposed a sharp lower bound on $\gamma_{d R}(G)$ in [12]. In [3], it was proved that $\gamma_{d R}(G)+2 \leqslant \gamma_{d R}(M(G)) \leqslant \gamma_{d R}(G)+3$, where $M(G)$ is the Mycielskian graph of $G$ and a construction was also given which confirms that there is no relation between the double Roman domination number of a graph and its induced subgraphs. The impact of some graph operations such as corona, cartesian product and addition of twins, on double Roman domination number was studied in [4]. In [2], J. Amjadi et al. improved the upper bound on $\gamma_{d R}(G)$ given in [6] by showing that for any connected graph $G$ of order $n$ with minimum degree at least two, $\gamma_{d R}(G) \leqslant \frac{8 n}{7}$.

\subsection{Basic Definitions and Preliminaries}

The open neighborhood of a vertex $v \in V$ is the set $N(v)=\{u: u v \in E\}$, and its closed neighborhood is $N[v]=N(v) \cup\{v\}$. The vertices in $N(v)$ are called the neighbors of $v$. When $G$ must be explicit, these open and closed neighborhoods are denoted by $N_{G}(v)$ and $N_{G}[v]$, respectively. $|N(v)|$ is called the degree of the vertex $v$ in $G$ and is denoted by $d_{G}(v)$, or simply $d(v)$. A vertex of degree 0 is known as an isolated vertex of $G$.

If $U$ is a non-empty subset of the vertex set $V$ of the graph $G$ then the sub- 
graph $\langle U>$ of $G$ induced by $U$ is defined as the graph having vertex set $U$ and edge set consisting of those edges of $G$ that have both ends in $U$. A subset $S$ of the vertex set $V$ of a graph $G$ is called independent if no two vertices of $S$ are adjacent in $G$. $S \subseteq V$ is a maximum independent set of $G$ if $G$ has no independent set $S^{\prime}$ with $\left|S^{\prime}\right|>|S|$. The number of vertices in a maximum independent set of $G$ is called the independence number, denoted by $\alpha(G)$. A complete graph on $n$ vertices, denoted by $K_{n}$, is the graph in which any two vertices are adjacent.

A Roman dominating function $(\mathrm{RDF})$ on a graph $G=(V, E)$ is defined as a function $f: V \rightarrow\{0,1,2\}$ satisfying the condition that every vertex $v$ for which $f(v)=0$ is adjacent to at least one vertex $u$ for which $f(u)=2$. The weight of a RDF is the value $f(V)=\sum_{v \in V} f(v)$. The Roman domination number of a graph $G$, denoted by $\gamma_{R}(G)$, is the minimum among the weights of RDFs on $G$.

Let $G=(V, E)$ be a non-empty graph of order $n \geqslant 2$, and $t$ a positive integer. We denote by $V^{t}$ the set of words of length $t$ on alphabet $V$. The letters of a word $u$ of length $t$ are denoted by $u_{1} u_{2} \ldots u_{t}$. Klavžar and Milutinović introduced in [9] the graph $S\left(K_{n}, t\right), t \geqslant 1,\left(S(t, n)\right.$ in their notation) whose vertex set is $V^{t}$, where $\{u, v\}$ is an edge if and only if there exists $i \in\{1,2, \ldots, t\}$ such that:

(i) $u_{j}=v_{j}$, if $j<i$; (ii) $u_{i} \neq v_{i}$; (iii) $u_{j}=v_{i}$ and $v_{j}=u_{i}$ if $j>i$.

Later, those graphs have been called Sierpiński graphs in [10]. This construction was generalized in [7] for any graph $G=(V, E)$, by defining the $t^{\text {th }}$ generalized Sierpiński graph of $G$, denoted by $S(G, t)$, as the graph with vertex set $V^{t}$ and edge set $\left\{\left\{w u_{i} u_{j}^{r-1}, w u_{j} u_{i}^{r-1}\right\}:\left\{u_{i}, u_{j}\right\} \in E, i \neq j ; r \in\{1,2, \ldots, t\} ; w \in V^{t-r}\right\}$. (See Figure 1 and 2,) Vertices of the form $x x \ldots x$ are called extreme vertices of $S(G, t)$. Note that for any graph $G$ of order $n$ and any integer $t \geqslant 2, S(G, t)$ has $n$ extreme vertices and, if $x$ has degree $d(x)$ in $G$, then the extreme vertex $x x \ldots x$ of $S(G, t)$ also has degree $d(x)$.

For any graph theoretic terminology and notations not mentioned here, the readers may refer to [5]. The following results are useful in this paper.

Proposition 1.1. [6] In a double Roman dominating function of weight $\gamma_{d R}(G)$, no vertex needs to be assigned the value 1 .

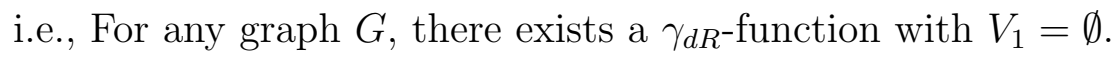



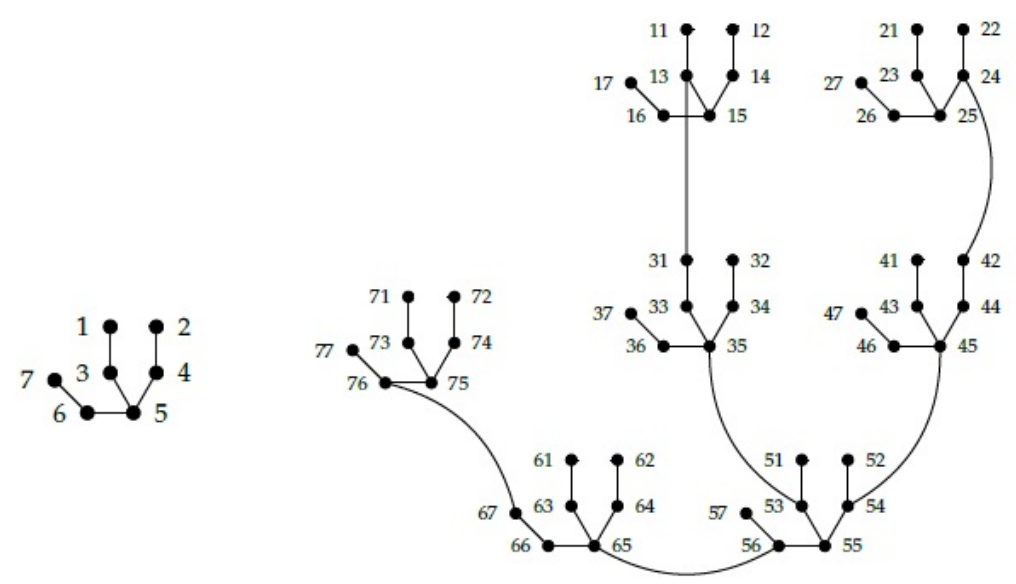

Figure 1: A graph $G$ and $S(G, 2)$.

Theorem 1.2. [11] For any integers $n \geqslant 2$ and $t \geqslant 1$,

$$
\gamma_{R}\left(S\left(K_{n}, t\right)\right) \leqslant\left\{\begin{array}{lll}
\frac{2 n^{t}+n-1}{n+1}, & t & \text { even } \\
\frac{2\left(n^{t}+1\right)}{n+1}, & t & \text { odd }
\end{array}\right.
$$

\section{Bounds on the Double Roman Domination Number}

First we prove a lower bound for $\gamma_{R}(S(G, t))$.

Theorem 2.1. For any graph $G$ of order $n, \gamma_{R}(S(G, t)) \geqslant n^{t-2} \alpha(G) \gamma_{R}(G)$, where $\alpha(G)$ is the independence number of $G$.

Proof. Let $V^{\prime} \subseteq V$ be an independent set of cardinality $\alpha(G)$. For any $w \in$ $V^{t-2}, i \in V$, let $V_{w i}=\{w i j: j \in V\}$. Note that $\left\{V_{w i}: i \in V\right\}$ is a partition of the vertex set of $S(G, t)$ and $\left\langle V_{w i}>\cong G\right.$, for every $i$ and hence there are $n^{t-1}$ disjoint copies of $G$ in $S(G, t)$. If $u$ and $v$ are adjacent in $S(G, t)$, then $u$ and $v$ are of the form $u=w x y^{r-1}, v=w y x^{r-1}$, where $w \in V^{t-r}, r \in\{1,2, \ldots, t\}$ and $x$ and $y$ are adjacent in $G$. Hence, for every $i, j \in V^{\prime}$, none of the vertices in $V_{w i}$ is adjacent to any of the vertices in $V_{w j}$. Also, $N\left(V_{w i}\right) \cap N\left(V_{w j}\right)=\emptyset$, for 


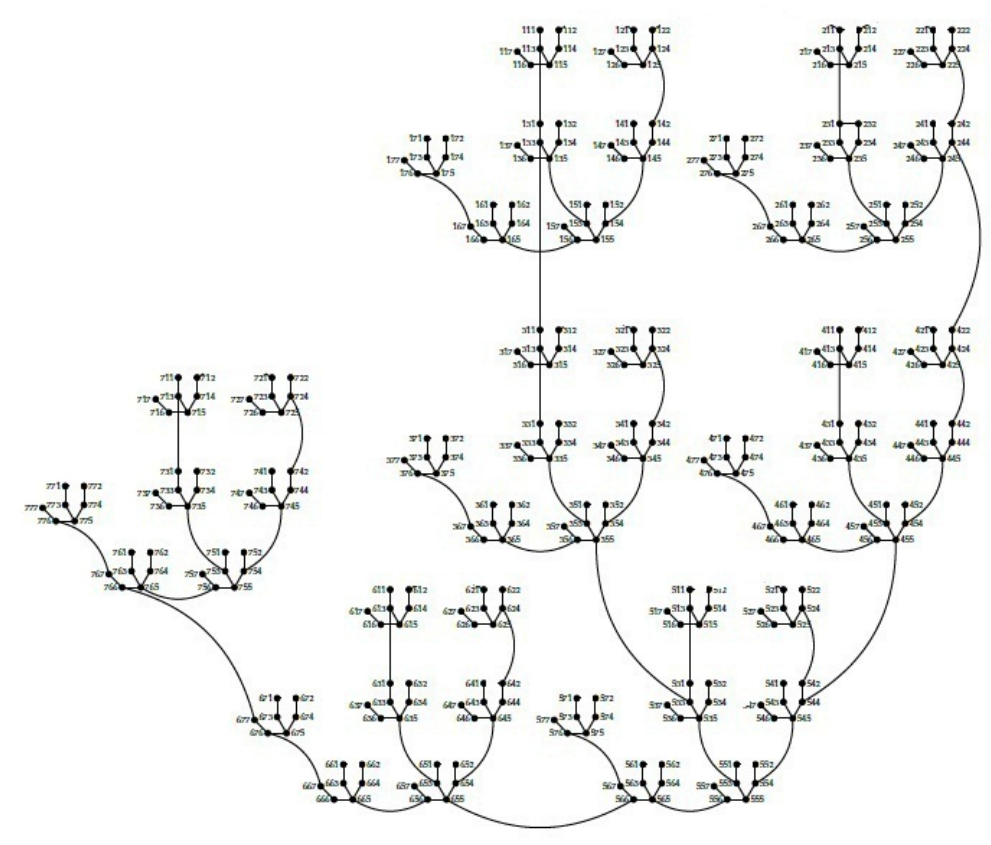

Figure 2: $S(G, 3)$ for the graph $G$ in Figure 1

$i \neq j$. Therefore, $f\left(V^{t}\right) \geqslant n^{t-2} \alpha(G) \gamma_{R}(G)$ for any RDF $f$ of $S(G, t)$ and hence $\gamma_{R}(S(G, t)) \geqslant n^{t-2} \alpha(G) \gamma_{R}(G)$.

Remark 2.1. It is clear that the inequality in Theorem 2.1 holds for other domination parameters like domination number, independence domination number, total domination number and many more.

Theorem 2.2. Let $G$ be a graph of order $n$. For any $\gamma_{d R}$-function $f=\left(V_{0}, V_{2}, V_{3}\right)$ on $G$, and any integer $t \geqslant 2$,

$$
n^{t-2} \alpha(G) \gamma_{d R}(G) \leqslant \gamma_{d R}(S(G, t)) \leqslant n^{t-2}\left(n \gamma_{d R}(G)-\left|V_{3}\right|-\left|D_{3}\right|\right),
$$

where $\alpha(G)$ is the independence number of $G$ and $D_{3}$ is the set of non-isolated vertices in $\left\langle V_{3}>\right.$. 
Proof. For the left inequality, the proof is as same as that of the Theorem 2.1.

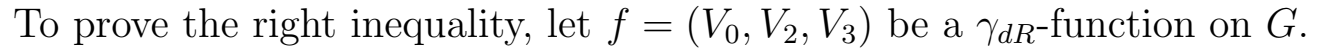

Step 1: For a given integer $t \geqslant 2$, let $S_{i}=\left\{w x: w \in V^{t-1}, x \in V_{i}\right\}$, for $i \in\{0,2,3\}$. Let $g: V^{t} \rightarrow\{0,2,3\}$ such that $g=\left(S_{0}, S_{2}, S_{3}\right)$. If $v \in V^{t}$ and $g(v)=0$, then $v=w y$ where $w$ is a word in $V^{t-1}$ and $y \in V_{0}$. Since $f$ is a $\gamma_{d R^{-}}$ function on $G$, there is either $z \in V_{3} \cap N_{G}(y)$ or $x_{1}, x_{2} \in V_{2} \cap N_{G}(y)$. Hence, there exists either $w z \in V_{3} \cap N_{S(G, t)}(w y)$ or $w x_{1}, w x_{2} \in V_{2} \cap N_{S(G, t)}(w y)$. Therefore, $g$ is a double Roman dominating function on $S(G, t)$ and $\gamma_{d R}(S(G, t)) \leqslant g\left(V^{t}\right)=$ $n^{t-1}\left(2\left|V_{2}\right|+3\left|V_{3}\right|\right)=n^{t-1} \gamma_{d R}(G)$.

Step 2: Let $S_{3}^{\prime}=\left\{w u u: w \in V^{t-2}, u \in V_{3}\right\}$. We define $g_{1}: V^{t} \rightarrow\{0,2,3\}$ such that $g_{1}=\left(S_{0}, S_{2} \cup S_{3}^{\prime}, S_{3}-S_{3}^{\prime}\right)$. Let $y \in S_{0}$. Then $y$ has the form

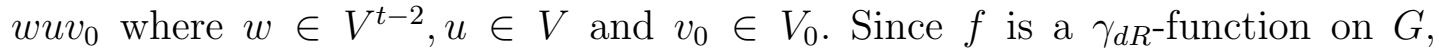
there is either $v_{3} \in V_{3}$ or $v_{2}, v_{2}^{\prime} \in V_{2}$ in $N_{G}\left(v_{0}\right)$. Therefore, there exists either $w u v_{3} \in N_{S(G, t)}\left(w u v_{0}\right)$ or $w u v_{2}, w u v_{2}^{\prime} \in N_{S(G, t)}\left(w u v_{0}\right)$. If $w u v_{3} \in S_{3}-S_{3}^{\prime}$, or $w u v_{2}, w u v_{2}^{\prime} \in N_{S(G, t)}\left(w u v_{0}\right)$, then we are done. Now, if $w u v_{3} \in S_{3}^{\prime}$, then $v_{3}=u$ and, since $v_{0}$ is adjacent to $v_{3}$, we can conclude that $y=w v_{3} v_{0}$ is adjacent to $w v_{0} v_{3} \in S_{3}-S_{3}^{\prime}$. Hence $g_{1}$ is a double Roman dominating function on $S(G, t)$. Therefore, $\gamma_{d R}(S(G, t)) \leqslant g_{1}\left(V^{t}\right) \leqslant n^{t-2}\left(n \gamma_{d R}(G)-\left|V_{3}\right|\right)$.

Step 3: Let $S_{3}^{\prime \prime}=\left\{w v v: w \in V^{t-2}, v \in D_{3}\right.$, where $D_{3}$ is the set of non isolated vertices in $\left.<V_{3}>\right\}$. We define $g_{2}: V^{t} \rightarrow\{0,2,3\}$ such that $g_{2}=\left(S_{0} \cup S_{3}^{\prime \prime}, S_{2} \cup S_{3}^{\prime}, S_{3}-S_{3}^{\prime}\right)$. Let $x \in V^{t}$ such that $g_{2}(x)=0$. In this case, $g_{1}(x)=0$ or $x \in S_{3}^{\prime \prime}$.

Suppose that $g_{1}(x)=0$. Then $x \in S_{0}$ and hence is of the form $x=w u v_{0}$, where $w \in V^{t-2}, u \in V$ and $v_{0} \in V_{0}$. If $N_{S(G, t)}(x) \cap S_{3}^{\prime \prime}=\emptyset$, then there exists $y \in N_{S(G, t)}(x) \cap\left(S_{3}-S_{3}^{\prime}\right)$ or $y_{1}, y_{2} \in N_{S(G, t)}(x) \cap\left(S_{2} \cup S_{3}^{\prime}\right)$. On the other side, if $z \in N_{S(G, t)}(x) \cap S_{3}^{\prime \prime}$, then $z=w v_{3} v_{3}$, where $v_{3} \in D_{3}, u=v_{3}$ and $v_{3}$ is adjacent to $v_{0}$, which implies that $x=w v_{3} v_{0}$ is adjacent to $w v_{0} v_{3}$ and we know that $g_{2}\left(w v_{0} v_{3}\right)=g_{1}\left(w v_{0} v_{3}\right)=g\left(w v_{0} v_{3}\right)=3$.

Now, if $x \in S_{3}^{\prime \prime}$, then $x=w v v$, where $w \in V^{t-2}$ and $v \in D_{3}$. So, by definition of $D_{3}, x$ must be adjacent to $w v u$ for some $u \in D_{3}-\{v\}$. Also, $g_{2}(w v u)=$ $g_{1}(w v u)=g(w v u)=f(u)=3$. Therefore, $g_{2}$ is a double Roman dominating function on $S(G, t)$, and so $\gamma_{d R}(S(G, t)) \leqslant n^{t-2}\left(n \gamma_{d R}(G)-\left|V_{3}\right|-\left|D_{3}\right|\right)$. 


\section{The Particular Case of Complete Graphs}

We begin this section by proving the Roman domination number of $S\left(K_{n}, 2\right)$.

Theorem 3.1. $\gamma_{R}\left(S\left(K_{n}, 2\right)\right)=2 n-1$.

Proof. By Theorem 1.2, we can easily deduce that $\gamma_{R}\left(S\left(K_{n}, 2\right)\right) \leqslant 2 n-1$. For the reverse inequality, let $V\left(K_{n}\right)=\left\{u_{1}, u_{2}, \ldots, u_{n}\right\}$. Then $S\left(K_{n}, 2\right)$ is a graph with vertex set $\left\{u_{i} u_{j}: i, j \in\{1,2, \ldots, n\}\right\}$ and edge set $\left\{\left\{u_{i} u_{j}, u_{j} u_{i}\right\}: u_{i}, u_{j} \in V, i \neq\right.$ $j\} \cup\left\{\left\{u_{i} u_{j}, u_{i} u_{k}\right\}: u_{i}, u_{j}, u_{k} \in V, j \neq k\right\}$. Note that $G_{i}=\left\{u_{i} u_{j}: j=1,2, \ldots, n\right\}$ induces a copy of $K_{n}$ for each $i=1,2, \ldots, n$ and vertex $u_{i} u_{i}$ is an extreme vertex of $S\left(K_{n}, 2\right)$ for each $i$. Let $f=\left(V_{0}, V_{1}, V_{2}\right)$ be any $\gamma_{R}$-function of $S\left(K_{n}, 2\right)$. Since $d_{S\left(K_{n}, 2\right)}\left(u_{i} u_{i}\right)=d_{K_{n}}\left(u_{i}\right)=n-1$, to Roman dominate the extreme vertex $u_{i} u_{i}, G_{i}$ must contain either a vertex of value 2 or $u_{i} u_{i}$ is of value 1 . If every $G_{i}$ contains a vertex of value 2 , then $f\left(V\left(S\left(K_{n}, 2\right)\right)\right) \geqslant 2 n$, which is a contradiction. Therefore, there exists at least one $G_{i}$, say $G_{i_{0}}$, which contains exactly one vertex in $V_{1}$ (and all other vertices are in $V_{0}$ ). Then, by the property of extreme vertex, the vertex in $V_{1}$ is $u_{i_{0}} u_{i_{0}}$. So, to Roman dominate $u_{i_{0}} u_{j}$, for each $j \neq i_{0}, u_{j} u_{i_{0}} \in V_{2}$. Therefore, $\gamma_{R}\left(S\left(K_{n}, 2\right)\right) \geqslant 2(n-1)+2=2 n-1$. Hence, the result.

Theorem 3.2. $\gamma_{d R}\left(S\left(K_{n}, 2\right)\right)=3 n-1$.

Proof. By Theorem 2.2, we can easily deduce that $\gamma_{d R}\left(S\left(K_{n}, 2\right)\right) \leqslant 3 n-1$, since $\left|V_{3}\right|=1$ and $\left|D_{3}\right|=0$. For the reverse inequality, let $V\left(K_{n}\right)=\left\{u_{1}, u_{2}, \ldots, u_{n}\right\}$. Then $S\left(K_{n}, 2\right)$ is a graph with vertex set $\left\{u_{i} u_{j}: i, j \in\{1,2, \ldots, n\}\right\}$ and edge set $\left\{\left\{u_{i} u_{j}, u_{j} u_{i}\right\}: u_{i}, u_{j} \in V, i \neq j\right\} \cup\left\{\left\{u_{i} u_{j}, u_{i} u_{k}\right\}: u_{i}, u_{j}, u_{k} \in V, j \neq k\right\}$. Note that $G_{i}=\left\{u_{i} u_{j}: j=1,2, \ldots, n\right\}$ induces a copy of $K_{n}$ for each $i=1,2, \ldots, n$ and vertex $u_{i} u_{i}$ is an extreme vertex of $S\left(K_{n}, 2\right)$ for each $i$. Let $f$ be any $\gamma_{d R^{-}}$ function of $S\left(K_{n}, 2\right)$. To double Roman dominate the extreme vertex $u_{i} u_{i}, G_{i}$ must contain either one vertex having value 3 , or two vertices having value 2 or $u_{i} u_{i}$ is of value 2 . If every $G_{i}$ contains a vertex of value 3 or two vertices of value 2 , then $f\left(V\left(S\left(K_{n}, 2\right)\right) \geqslant 3 n\right.$ which is a contradiction to Theorem 2.2. Therefore, there exists at least one $G_{i}$, say $G_{i_{0}}$, which contains exactly one vertex having value 2 . Then, by the property of extreme vertex the value 2 is assigned to $u_{i_{0}} u_{i_{0}}$. To double Roman dominate $u_{i_{0}} u_{j}$, for each $j \neq i_{0}, u_{j} u_{i_{0}}$ must be in $V_{2} \cup V_{3}$. If $u_{j} u_{i_{0}} \in V_{2}$, then $G_{j}$ contains at least one more vertex of value at least 2 . Therefore, it is optimal to assign the value 3 to $u_{j} u_{i_{0}}$, for every $j \neq i_{0}$. But then $f\left(V\left(S\left(K_{n}, 2\right)\right) \geqslant 2+3(n-1)\right.$. Hence, $\gamma_{d R}\left(S\left(K_{n}, 2\right)\right)=3 n-1$.

Acknowledgements: The first author thanks University Grants Commission for granting fellowship under Faculty Development Programme (F.No.FIP $/ 12^{\text {th }}$ plan/KLMG 045 TF 07 of UGC-SWRO). 


\section{References}

[1] H. A. Ahangar, M. A. Henning, V. Samodivkin, I. G. Yero, Total Roman Domination in Graphs, Appl. Anal. Discrete Math., 10 (2016), 501-517.

[2] J. Amjadi, S. Nazari-Moghaddam, S. M. Sheikholeslami, L. Volkmann, An Upper Bound on the Double Roman Domination Number, J. Comb. Optim., (to appear).

[3] Anu V., Aparna Lakshmanan S., Double Roman Domination Number, Discrete Appl. Math., 244 (2018), 198-204.

[4] Anu V., Aparna Lakshmanan S., Impact of Some Graph Operations on Double Roman Domination Number, (Submitted), (2018).

[5] R. Balakrishnan, K. Ranganathan, A Text Book of Graph Theory, Springer, New York, (1999).

[6] R. A. Beeler, T. W. Haynes, S. T. Hedetniemi, Double Roman Domination, Discrete Appl. Math., 211(1) (2016), 23-29.

[7] S. Gravier, M. Kovše, A. Parreau, Generalized Sierpiński Graphs, in: Posters at EuroComb'11, Rényi Institute, Budapest, URL http://www.renyi.hu/conferences/ec11/posters/parreau.pdf, (Website visited last on 21.12.2018), (2011).

[8] G. Hao, X. Chen, L. Volkmann, Double Roman Domination in Digraphs, Bull. Malays. Math. Sci. Soc., (to appear).

[9] S. Klavžar, U. Milutinović, Graphs $S(n, k)$ and a Variant of the Tower of Hanoi Problem, Czechoslovak Math. J., 47 (1) (1997), 95-104.

[10] S. Klavžar, U. Milutinović, C. Petr, 1-Perfect Codes in Sierpinski Graphs, Bull. Austral. Math. Soc., 66 (3) (2002), 369-384.

[11] F. Ramezani, E. D. Rodríguez-Bazan, J. A. Rodríguez-Velázquez, On the Roman Domination Number of Generalized Sierpiński Graphs, Filomat, 31(20) (2017), 6515-6528.

[12] L. Volkmann, Double Roman Domination and Domatic Numbers of Graphs, Commun. Comb. Optim., 3(1) (2018), 71-77. 